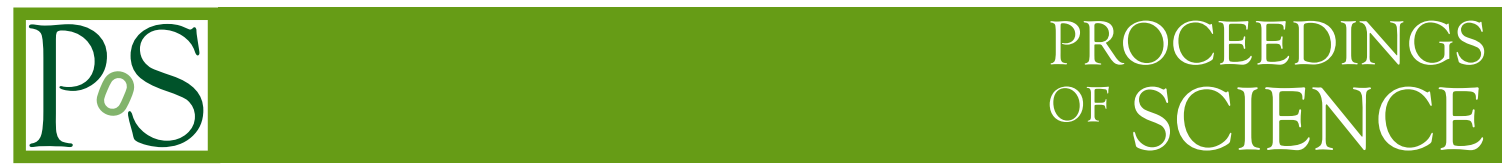

\title{
Event plane determination with the new ALICE FIT detector
}

\section{Heidi Rytkönen ${ }^{a}$ on behalf of the ALICE collaboration}

${ }^{a}$ Department of Physics, University of Jyväskylä, PO Box 35, FI-40014 University of Jyväskylä, Finland

E-mail: heidi.rytkonen@cern.ch

During the on-going second long shutdown of LHC, the forward detectors of the ALICE experiment are implementing an extensive upgrade. In particular, a new Fast Interaction Trigger (FIT) has been designed and built. It consists of three sub-detector systems delivering a broad range of online functionalities, and an essential input for event characterization and physics analysis. For instance, FIT will deliver the precise collision time for the TOF-based particle identification, provide the centrality and the event plane information, and measure the cross section of diffractive processes. This note will discuss usage of FIT in the event plane determination during Run 3. A simulated event plane resolution at $\sqrt{\mathrm{s}_{\mathrm{NN}}}=2.76 \mathrm{TeV}$ is also presented.

40th International Conference on High Energy physics - ICHEP2020

July 28 - August 6, 2020

Prague, Czech Republic (virtual meeting) 


\section{Introduction}

Quark-gluon plasma (QGP), a state of deconfined and strongly interacting matter existing at high temperatures and densities, is the main subject of study in ultra-relativistic heavyion collisions. ALICE is the only dedicated heavy-ion experiment at the LHC. During the on-going second long shutdown of the CERN accelerator system, ALICE is upgrading several of its detectors and installing three new ones [1].

One of the new detectors is the Fast Inter-

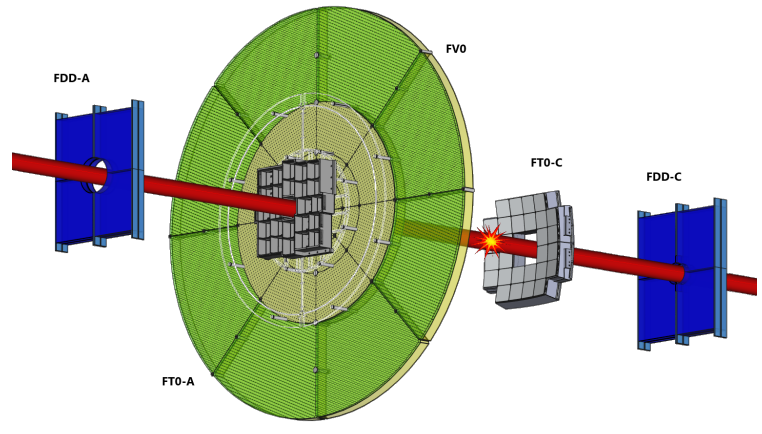

Figure 1: The active parts of the three FIT subsystems. action Trigger (FIT) [2], shown in Figure 1. FIT consists of two Cherenkov arrays called FT0 on both sides (A- and C-side) of the interaction point, as well as two scintillation detectors at high pseudorapidities that make up the Forward Diffractive Detector (FDD). FT0-A is attached to FV0, a large scintillation disk. The two other additions to ALICE are the new Inner Tracking System (ITS) and the Muon Forward Tracker (MFT).

FIT has several functionalities [2] such as luminosity monitoring for both ALICE and LHC, trigger generator, and the collision time measurement needed for the particle identification. Also, FIT subsystems are used for centrality and event plane determination. Presently we are studying the performance of the upgraded forward detectors.

\section{Event plane determination}

The reaction plane, $\Psi_{\mathrm{RP}}$, of a heavy-ion collision is defined by the beam axis and impact parameter vector connecting the centers of the colliding nuclei. Since the impact parameter cannot be measured in real experiments, one has to estimate $\Psi_{\mathrm{RP}}$ from observed final state particles. The azimuthal distribution of the final state particles can be written

$$
\frac{\mathrm{d} N}{\mathrm{~d} \phi} \propto 1+\sum_{n=1}^{\infty} 2 v_{n} \cos \left(n\left(\phi-\Psi_{n}\right)\right),
$$

where $v_{n}$ 's are the flow Fourier coefficients (flow harmonics) and $\Psi_{n}$ 's are the so-called event plane angles [3]. As the second flow harmonic $v_{2}$ is the most directly related to the geometry of the heavy-ion collision and enhances the particle emission in the in-plane direction, we use the second-order event plane as an estimate for the reaction plane.

The event plane angles $\Psi_{n}$ are determined, event-by-event, using a Q-vector [3]. The Q-vector components are calculated by summing over each particle in the event in the following way:

$$
\vec{Q}_{n}=\left(\sum_{i} \cos \left(n \phi_{i}\right), \sum_{i} \sin \left(n \phi_{i}\right)\right) .
$$

Here $\phi_{i}$ is the azimuthal angle of the particle, and $n$ refers to the flow harmonic. Using the components of $\vec{Q}_{2}$, the second order event plane is then

$$
\Psi_{2}=\arctan \left(\frac{Q_{2, x}}{Q_{2, y}}\right) / 2 .
$$


Due to the finite multiplicity of the collision, detector effects, and secondary particles that might obscure the flow signal, the event plane differs from the real reaction plane. This difference is quantified by the event plane resolution

$$
R_{2}=\left\langle\cos \left(2\left(\Psi_{2}-\Psi_{\mathrm{RP}}\right)\right)\right\rangle,
$$

where the mean $\langle\cdots\rangle$ is calculated over all the events. As the true reaction plane $\Psi_{\mathrm{RP}}$ is unknown, the resolution is estimated using various methods involving correlations between the event planes obtained with different detectors [3].

\section{Measuring event plane with FIT}

The event plane resolution of various FIT subsystems has been estimated previously at $2.76 \mathrm{TeV}$ via simulations, see Figure 2. At the moment the event plane resolution is studied at 5.5 TeV that is the energy for $\mathrm{Pb}-\mathrm{Pb}$ collisions during Run 3. The initial collision is simulated using AMPT (A Multi-Phase Transport Model) generator [4] that is capable of producing the needed flow signal [5], and the particle transport through the simulated ALICE detector setup is done using GEANT.

In these simulations, we aim to understand, for example, the effect of the secondaries on the resolution in the new upgraded detector setup. Also, the event plane needs several corrections [3] once the real data is measured.

\section{Summary}

The expected performance of the FIT detector matches all ALICE requirements for the LHC Run 3 and 4. Currently, in preparation for the physics analysis of the first collision data, we are conducting detailed simulation studies for the event plane determination. They are needed for the error analysis and the calibration.

\section{References}

[1] W. H. Trzaska, Nucl. Instrum. Methods Phys. Res. A 958(2020) 162116.

[2] W. H. Trzaska, Nucl. Instrum. Methods Phys. Res. A 845:463-466 (2017).

[3] A. M. Poskanzer, and S.A. Voloshin, Phys. Rev. C, 58:1671-1678 (1998).

[4] Z. W. Lin, C. M. Ko, B. A. Li, B. Zhang, and S. Pal, Phys. Rev. C 72 (2005) 064901.

[5] S.Acharya et al. [ALICE], Phys. Rev. C 97 (2018) no.2, 024906. 